A didactic experiment and model of a flat-plate solar collector

This article has been downloaded from IOPscience. Please scroll down to see the full text article. 2011 Phys. Educ. 46312

(http://iopscience.iop.org/0031-9120/46/3/010)

View the table of contents for this issue, or go to the journal homepage for more

Download details:

IP Address: 147.163.21.165

The article was downloaded on 05/05/2011 at 08:38

Please note that terms and conditions apply. 


\title{
A didactic experiment and model of a flat-plate solar collector
}

\author{
Aurelio Agliolo Gallitto ${ }^{1}$ and Emilio Fiordilino \\ Dipartimento di Fisica, Università di Palermo, via Archirafi 36, I-90123 Palermo, Italy \\ E-mail: agliolo@fisica.unipa.it
}

\begin{abstract}
We report on an experiment performed with a home-made flat-plate solar collector, carried out together with high-school students. To explain the experimental results, we propose a model that describes the heating process of the solar collector. The model accounts quantitatively for the experimental data. We suggest that solar-energy topics should be included in school programmes to give students the opportunity to gain experience with solar energy and increase their awareness of the benefits that can be obtained from this remarkable and renewable energy source.
\end{abstract}

\section{Introduction}

Since prehistory, mankind has always recognized the Sun as a vital source of energy. As an example, figure 1 shows a picture of a 5000-yearold piece of pottery with an engraved image of the Sun. However, only in much more recent years did investigation on solar energy begin. In the eighteenth century, the Swiss physicist Horace de Saussure (1740-1799) studied how to exploit solar energy to warm a small container. He built a 'miniature greenhouse' made of wood with glass covers that was able to trap the energy coming from the Sun. He placed five glass boxes, stacked one inside the other, on a black wooden table. To improve thermal insulation, he placed a wooden box in the middle of an open-topped container and stuffed wool packing between the sides of the container and the walls of the box. In this way, the temperature in the hot box rose up to $110^{\circ} \mathrm{C}$ [1]. Subsequently, other scientists studied the effects of solar energy and performed experiments with hot boxes; they demonstrated that temperatures exceeding the boiling point of water could be

\footnotetext{
1 Author to whom any correspondence should be addressed.
}

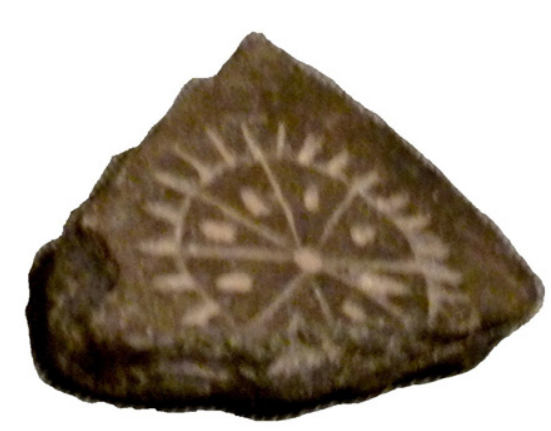

Figure 1. An archaeological find with an engraved image of the sun, on display at the National Museum of Valletta, Malta [2].

reached in a glass-covered box under sunlight. The hot box became the prototype for the modern flatplate solar collectors, which can supply houses with hot water, heat and air conditioning.

The inclusion of solar-energy topics in school programmes could give students the possibility of gaining experience with energy-supply systems and could raise their awareness of the benefits that can be obtained in daily life by using this remarkable and renewable energy source. 
(a)

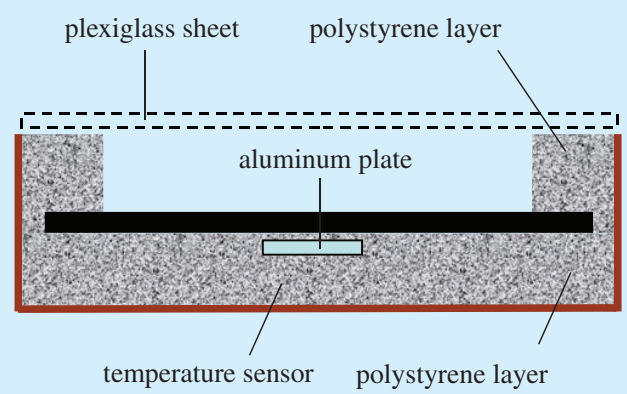

Figure 2. (a) A draft of the wooden box used in the experiment. (b) Experimental apparatus consisting of a wooden box, an incandescent lamp and an electronic thermometer. (b)

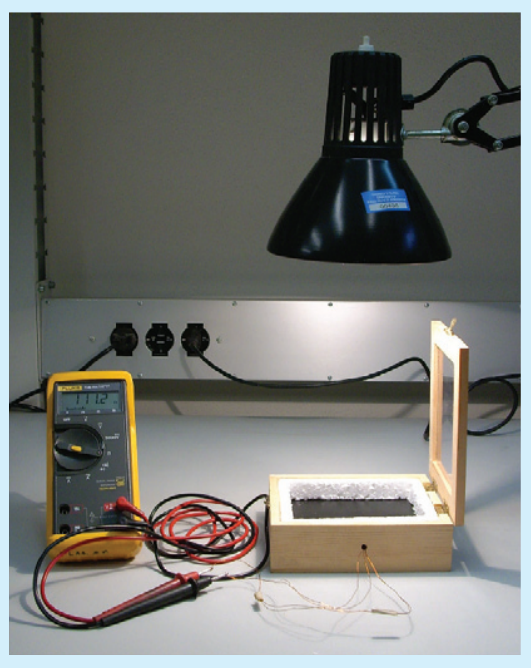

In this article, we report an experiment on a home-made flat-plate solar collector carried out together with high-school students, as part of the Italian National Project 'Lauree Scientifiche' [2-4]. Here, we propose a model to describe the heating process of the solar collector.

\section{Experimental apparatus}

To perform the experiment, we made a wooden box of dimensions $16 \times 13.5 \times 4.5 \mathrm{~cm}^{3}$ having walls insulated by panels of expanded polystyrene about $2 \mathrm{~cm}$ thick (a recycled cardboard box would also be suitable for the purpose). On the bottom of the box we located a black-painted aluminium sheet about $0.5 \mathrm{~mm}$ thick and $15 \times 12.5 \mathrm{~cm}^{2}$ in area. The box was used without a cover and was covered by a plexiglass sheet about $1.5 \mathrm{~mm}$ thick. The temperature inside the box was measured by a PT100 temperature sensor (it gives $R=100 \Omega$ at $T=0{ }^{\circ} \mathrm{C}$ ) located below the aluminium sheet. A draft of the box is shown in figure 2(a); the apparatus used for the indoor experiment is shown in figure 2(b).

The experiment should be performed with solar insolation; however, this is not always possible during regular lessons at school. Therefore, for pedagogical reasons we suggest doing the experiment indoors using an incandescent lamp as the source of light. The collector was then exposed to the light emitted by a $60 \mathrm{~W}$ incandescent lamp and the temperature inside the box was recorded as a function of time. The experiment was performed

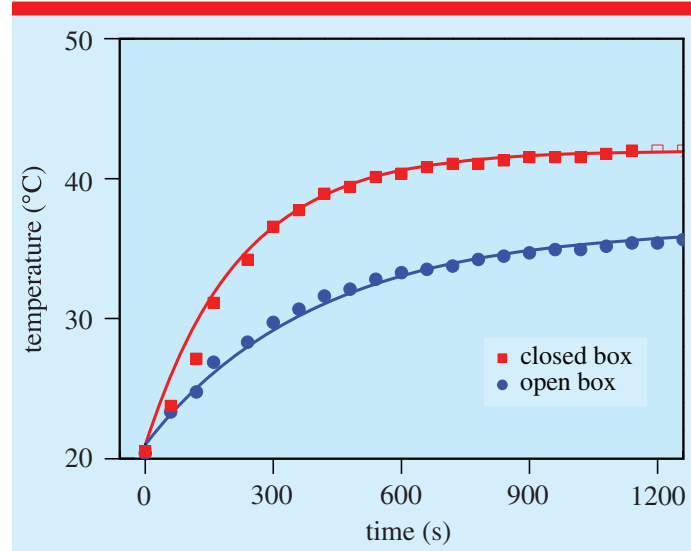

Figure 3. Temperature versus time measured inside the illuminated box with and without plexiglass cover. Symbols are experimental data; continuous lines are expected curves obtained, as explained in the text.

at room temperature $T_{0} \approx 20^{\circ} \mathrm{C}$ with the box uncovered and subsequently with the box covered by the plexiglass sheet; typical results are shown in figure 3. A temperature of about $36.5^{\circ} \mathrm{C}$ was reached for the uncovered box and about $42^{\circ} \mathrm{C}$ was attained when the plexiglass sheet covered the box.

Although the experiment takes about $20 \mathrm{~min}$ for each set of measurements, it is very convenient to use a data-logger system to automatically collect the values of temperature as a function of time. For this purpose, one can use the 
temperature sensor Go!Temp from Vernier ${ }^{2}$, which can be connected directly to a computer through the USB port; the Vernier company also provides the program Logger Lite that allows one to record and visualize the collected data.

\section{Theoretical description}

The Sun, through thermonuclear processes, generates thermal energy and electromagnetic (em) radiation with wavelength $\lambda$ ranging from ultraviolet $(\lambda \lesssim 380 \mathrm{~nm})$ to infrared $(\lambda \gtrsim 700 \mathrm{~nm})$; the visible region is for $380 \mathrm{~nm} \lesssim \lambda \lesssim 700 \mathrm{~nm}$. Solar radiation received at the ground is substantially in the range of $300-2500 \mathrm{~nm}$ and its average intensity is about $1 \mathrm{~kW} \mathrm{~m}^{-2}$, at sea level; about $460 \mathrm{~W} \mathrm{~m}^{-2}$ is provided by infrared radiation, $470 \mathrm{~W} \mathrm{~m}^{-2}$ by visible light and $70 \mathrm{~W} \mathrm{~m}^{-2}$ by ultraviolet radiation [5]. Fortunately, most of the harmful ultraviolet radiation is absorbed by the ozone layer in the stratosphere [6].

Electric current heats the filament of a lamp up to temperatures high enough to make it incandescent and to emit em radiation. The important point to be stressed here is that the Sun and all the incandescent lamps emit light with a spectrum that very closely resembles that of a black body. For this reason, the emission spectrum of both the Sun and the incandescent lamp can be described by the law of black-body emission. This makes reasonable our suggestion to use an incandescent lamp when direct sunlight is not available.

The properties of the em radiation are most conveniently described by providing the spectrum of the radiation, i.e. its wavelength (or frequency) content. Both the emission of the Sun and an incandescent lamp are very well accounted for by a black-body spectrum (of course, at different temperatures). The power per unit of surface and per unit of wavelength $R(\lambda)$ emitted by a black body is given by Planck's law [7]

$$
R(\lambda)=\frac{2 \pi h c^{2}}{\lambda^{5}} \frac{1}{\mathrm{e}^{\frac{h c}{\lambda k T}}-1},
$$

where $T$ is the temperature in kelvin $(\mathrm{K}), \lambda$ the wavelength, $c$ the speed of the light $(c=3 \times$ $\left.10^{8} \mathrm{~m} \mathrm{~s}^{-1}\right), h$ the Planck constant $(h=6.63 \times$ $\left.10^{-34} \mathrm{~J} \mathrm{~s}\right)$ and $k$ is the Boltzmann constant $(k=$ $1.38 \times 10^{-23} \mathrm{~J} \mathrm{~K}^{-1}$ ).

${ }^{2}$ See Vernier's website: www.vernier.com.

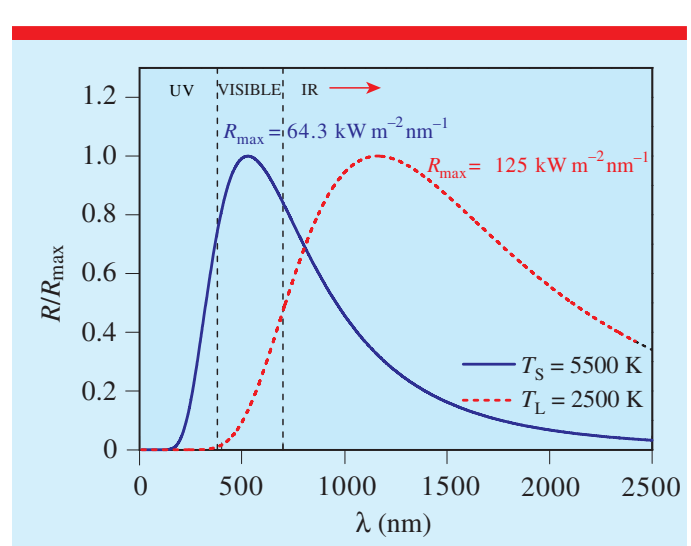

Figure 4. The normalized spectrum of the radiation emitted by the Sun at the temperature $T_{\mathrm{S}}=5500 \mathrm{~K}$ and by an incandescent lamp at the temperature $T_{\mathrm{L}}=2500 \mathrm{~K}$. The plot shows the power per unit of surface emitted by the black body in the wavelength range $(\lambda, \lambda+d \lambda)$ as a function of the wavelength $\left(1 \mathrm{~nm}=10^{-9} \mathrm{~m}\right)$.

The values of the peak wavelength $\lambda_{\max }$ and of the peak of $R_{\max }(T)$ are given, respectively, by Wien's laws

$$
\lambda_{\max }=\frac{h c}{4.97 k T}
$$

and

$$
R_{\max }(T)=\frac{2 \pi}{h^{4} c^{3}} \frac{(4.97 k T)^{5}}{e^{4.97}-1} .
$$

For didactic purposes, we describe the em radiation in terms of photons, with zero (rest) mass and zero electric charge. The energy of the photon is $E=h v=h c / \lambda$, where $v$ is the frequency.

In figure 4 , we show the normalized spectrum of the solar radiation $R(\lambda)$, calculated by considering the Sun as a black body at temperature $T_{\mathrm{S}}=5500 \mathrm{~K}$, and the normalized spectrum of the emitted radiation of the inert-gas-filled incandescent lamp, calculated by considering the lamp as a black body at temperature $T_{\mathrm{L}}=2500 \mathrm{~K}$ (according to the manufacturer's data sheets). The choice to normalize the spectra is due to the fact that the values of $R_{\max }$ for the Sun and for the lamp are very different.

When photons strike the opaque surface of a body, they are either absorbed or reflected. When absorbed, photons release their energy, which will be converted into thermal energy of the body. The rate of rise of the body temperature depends on its thermal capacity, the intensity of 


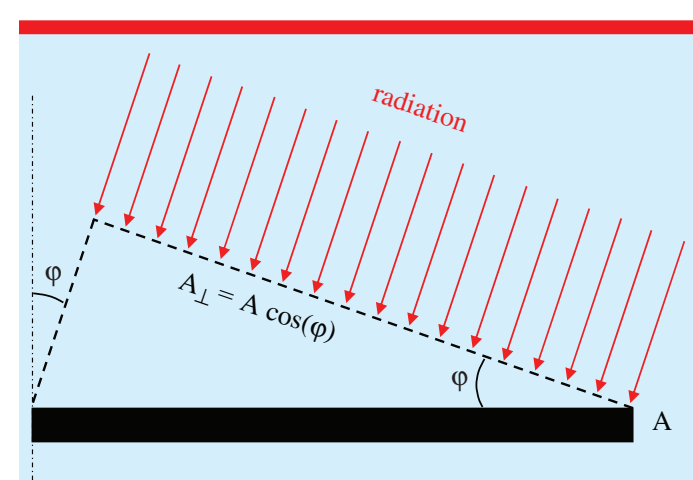

Figure 5. Effective area of an illuminated body.

the incident radiation and the heat leakage towards the environment. If the ambient temperature does not differ appreciably from that of the collector, it can be assumed that the available energy is approximately equal to the energy of the incoming em radiation; therefore, one may suppose that the energy $\mathrm{d} Q_{\mathrm{a}}$ absorbed under a constant illumination, in the time interval $\mathrm{d} t$, is given by

$$
\mathrm{d} Q_{\mathrm{a}}=\left(\eta I A_{\perp} \alpha\right) \mathrm{d} t,
$$

where $\alpha$ is the absorptance ${ }^{3}$; $I$ is the intensity of the radiation; $A$ is the effective area perpendicular to the incident radiation (as shown in figure 5, for a flat surface, the effective area $A_{\perp}$ is obtained by multiplying the area $A$ of the illuminated section by $\cos \varphi$, with $\varphi$ the incidence angle of the em radiation); $\eta$ is a coefficient of proportionality that takes into account the efficiency of the system.

We can suppose that on increasing the temperature $T$ of the box, heat starts flowing into the environment proportionally to the difference of temperature $T-T_{0}$, where $T_{0}$ is the temperature of the environment; it contributes negatively to the heating process of the box. The emitted energy transfers to the environment following Newton's law of cooling, that is

$$
\mathrm{d} Q_{\mathrm{e}}=-\frac{C\left(T-T_{0}\right)}{\tau} \mathrm{d} t,
$$

\footnotetext{
3 The absorptance $\alpha$ of a material is the ratio of energy absorbed by the material to energy absorbed by a black body at the same temperature. It is a measure of a material's ability to absorb energy. A true black body would have $\alpha=1$ while any real object has $\alpha<1$. Generally, $\alpha$ depends on the frequency; however, here we shall not take into account this dependency.
}

where $C$ is the thermal capacity of the body and $\tau$ a time constant characteristic of the dynamic system.

The time constant $\tau$ depends mainly on the shape of the body, the nature of the cooling fluid (air or liquid), which transports heat from the hot body towards the environment, and in the case of large temperature variations it depends also on the temperature.

The total exchanged energy is obtained by summing equations (4) and (5)

$\mathrm{d} Q=\mathrm{d} Q_{\mathrm{a}}+\mathrm{d} Q_{\mathrm{e}}=\left(\eta I A_{\perp} \alpha\right) \mathrm{d} t-\frac{C\left(T-T_{0}\right)}{\tau} \mathrm{d} t$.

By setting $\mathrm{d} Q=C \mathrm{~d} T$, the equation for the time dependence of the temperature $T$ becomes

$$
\mathrm{d} T=-\frac{\left(T-T_{0}-\theta\right)}{\tau} \mathrm{d} t,
$$

with $\theta \equiv \eta I A_{\perp} \tau \alpha / C$.

This equation is readily solved:

$$
\frac{\mathrm{d} T}{T-T_{0}-\theta}=-\frac{\mathrm{d} t}{\tau} ;
$$

integrating between $t=0$ and $t$ and between $T=T_{0}$ and $T=T(t)$, we obtain

$$
\ln \frac{T-T_{0}-\theta}{-\theta}=-\frac{t}{\tau}
$$

or

$$
T(t)-T_{0}=\theta\left(1-\mathrm{e}^{-t / \tau}\right) .
$$

Calling $T_{\infty}$ the temperature reached after a long time, the previous equation gives $\theta=T_{\infty}-T_{0}$ and equation (10) results in

$$
T(t)-T_{0}=\left(T_{\infty}-T_{0}\right)\left(1-\mathrm{e}^{-t / \tau}\right) .
$$

For large values of $t$, the temperature of the box reaches the limit temperature $T_{\infty}$; this condition is attained when the heat flowing out of the box is equal to the heat coming from irradiation, and no further temperature increase takes place. For small values of $t$, the temperature increases linearly with time as

$$
T(t)-T_{0}=\frac{\left(T_{\infty}-T_{0}\right)}{\tau} t=\frac{\eta I A_{\perp} \alpha}{C} t .
$$

Continuous lines in figure 3 are the curves of temperature as a function of time obtained by equation (11) using the following parameters: $T_{\infty}=42^{\circ} \mathrm{C}$ and $\tau=200 \mathrm{~s}$ for the covered box; $T_{\infty}=36.5^{\circ} \mathrm{C}$ and $\tau=400 \mathrm{~s}$ for the uncovered box. 


\section{Discussion and conclusion}

The measurements performed with covered and uncovered boxes show that the heating effects are more enhanced in the case of a covered box, because of the reduced convective hot-air streams, which transfer heat into the environment. The em radiation penetrates the plexiglass cover of the box and is absorbed by the aluminium black surface. In the process, the em energy coming from the Sun heats the box. The plexiglass cover allows radiation to pass through, thus heating the air inside the box, but it inhibits convective heat transfer towards the outside. However, some heat is still lost by conduction through the glass and the walls of the box. Although, plexiglass absorbs some infrared radiation [8], we prefer to use this material instead of glass mainly for safety reasons. The black colour of the aluminium plate maximizes the absorption of visible and infrared light because it behaves like a black body with $\alpha \lesssim 1$.

Although the experiment does not allow us to determine exactly the amount of energy collected in the box (as internal energy), it shows the heating effect of the em radiation. We can roughly estimate the power transferred to the aluminium plate by multiplying the initial slope of the temperature-versus-time curve of figure 3 by the thermal capacity of the aluminium [9]. The thermal capacity is calculated from the mass of the plate $(\approx 25 \mathrm{~g})$ and the specific heat of aluminium $\left(\approx 910 \mathrm{~J}\left(\mathrm{~kg}^{\circ} \mathrm{C}\right)^{-1}\right)$. In our case, the slope at $t=0$ of the temperature-versus-time curve of figure 3 for the closed box is about $0.083{ }^{\circ} \mathrm{C} \mathrm{s}^{-1}$. With these data, we obtain an absorbed power of about $2 \mathrm{~W}$.

We have found that the main problem for obtaining high temperatures inside our solar collector is the thermal insulation of the walls of the box to prevent energy exchange with the environment. Of course, this is an important factor to be considered also in civil engineering and architecture. Cellulose and mineral wool are the traditional and cheap insulation materials; today, modern plastic foams are more and more often used. These materials can be used in different ways (walls, ceilings, floors, etc) and have different degrees of effectiveness. A discussion of the performances of these materials goes beyond the scope of this article; we refer the interested reader to specialized books on the subject [10].
In conclusion, we have performed an experiment on a home-made flat-plate solar collector, carried out together with high-school students, aimed at exploiting solar energy to produce heat. We have proposed a model that describes the heating process of the solar collector. We would like to stress that although the model is valid when $T$ is not much larger than $T_{0}$ it accounts quite well for the experimental data, within the experimental uncertainty. From a pedagogical point of view, this feature should not be overlooked; the feasibility of the experiment is rather simple and allows the teacher to show the importance of a quantitative description of the experiment. In fact, it is important to stress even in high school that an experiment can be fully understood only when a quantitative description is provided.

The mathematical model here discussed can also be used to explain other phenomena in nature, which behave in a similar fashion. For example, the motion of a falling body in a viscous medium under the action of the force of gravity. In this case, the velocity of the falling body plays the same role as the temperature of the lighted box, described by the time dependence of the temperature given by equation (11), which is a general solution for many dynamic systems.

Finally, we suggest that solar-energy topics should be included in school programmes to give students the chance to gain experience with solar energy and increase their awareness of the benefits that can be obtained from this remarkable and renewable energy source.

\section{Acknowledgments}

This work was carried out in the framework of the Italian National Project 'Lauree Scientifiche' under the financial support of the Italian Ministry of Education, University and Research.

Received 21 January 2011, in final form 31 January 2011 doi:10.1088/0031-9120/46/3/010

\section{References}

[1] The Solar Cooking Archive-Horace de Saussure and his Hot Boxes of the 1700s http://solarcooking.org/saussure.htm

[2] Fiordilino E and Agliolo Gallitto A $2010 \mathrm{Il}$ laboratorio di fisica nel Progetto Lauree Scientifiche (Rome: Aracne)

[3] Agliolo Gallitto A and Fiordilino E 2007 Progetto Lauree Scientifiche-Fisica dell'Ateneo di Palermo Università e Scuola XII 1 39-42 
[4] Agliolo Gallitto A 2009 Quattro Anni di Attività del Progetto Lauree Scientifiche-Fisica nell'Ateneo di Palermo Università e Scuola XIV 2 28-33

[5] Duffie J A and Beckman W A 1974 Solar Energy Thermal Processes (New York: Wiley)

[6] Harrison T and Shallcoss D 2010 A hole in the sky Sci. Sch. 17 46-53

[7] Krane K S 1996 Modern Physics 2nd edn (New York: Wiley)

[8] Asdrubali F, Buratti C, Mariani R, Baldinelli G and Moretti E Misure spettrofotometriche su materiali trasparenti: recenti attività di ricerca del laboratorio di termotecnica dell'Università di Perugia Quaderno N.22, Collana Tecnico-Scientifica directed by G Galli, CIRIAF, Perugia, August 2004

[9] Vella G J and Goldsmid H J 1976 Simple experiments on the use of solar energy Phys. Educ. 11 413-6
[10] Kalogirou S 2009 Solar Energy Engineering: Processes and Systems (New York: Academic)

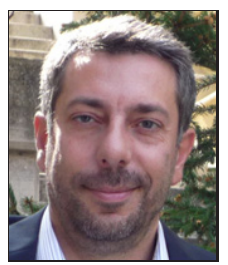

Aurelio Agliolo Gallitto is an associate professor of experimental physics at the University of Palermo, Italy and works in the field of superconductivity at microwave frequencies. From 2008 to 2010, he was the local coordinator of the national project 'Lauree Scientifiche'.

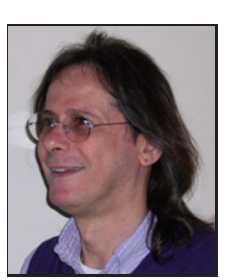

Emilio Fiordilino teaches field theory and statistical mechanics at the

University of Palermo, Italy. His research activity is mainly in the physics of the interaction of strong electromagnetic fields with molecules and nanoparticles, but also in volcanism. He spends part of his time in lecturing on quantum mechanics in high schools. 